\title{
Radial 3D-Needlets on the Unit Ball
}

\author{
Claudio Durastanti ${ }^{1}$, Yabebal T. Fantaye ${ }^{2}$, Frode K. Hansen ${ }^{3}$, \\ Domenico Marinucci ${ }^{4}$ and Isaac Z. Pesenson ${ }^{5}$ \\ ${ }^{1}$ Department of Mathematics, University of Tor Vergata, Rome, \\ email: durastan@mat. uniroma2.it \\ ${ }^{2}$ Department of Mathematics, University of Tor Vergata, Rome, \\ email: fantaye@mat.uniroma2.it \\ ${ }^{3}$ Institutt for teoretisk astrofysikk, University of Oslo, \\ email: f.k.hansen@astro.uio.no \\ ${ }^{4}$ Department of Mathematics, University of Tor Vergata, Rome, \\ email: marinucc@mat . uniroma2.it \\ ${ }^{5}$ Department of Mathematics, Temple University, Philadelphia, \\ email: isaak.pesenson@temple.edu
}

\begin{abstract}
We present a simple construction of spherical wavelets for the unit ball, which we label Radial 3D Needlets. We envisage an experimental framework where data are collected on concentric spheres with the same pixelization at different radial distances from the origin. The unit ball is hence viewed as a tensor product of the unit interval with the unit sphere: a set of eigenfunctions is therefore defined on the corresponding Laplacian operator. Wavelets are then constructed by a smooth convolution of the projectors defined by these eigenfunctions. Localization properties may be rigorously shown to hold in the real and harmonic domain, and an exact reconstruction formula holds; the system allows a very convenient computational implementation.
\end{abstract}

Keywords. methods: data analysis, methods: statistical, cosmology: observations

\section{Motivations and background}

It is well-known that Cosmology has recently experienced a golden era where datasets of unprecedented accuracy have become available, for instance on Cosmic Microwave Background radiation (see for instance Bobin et al. (2013), Planck XXIII (2013), and the references therein). These datasets are typically collected over the full-sky, usually covering thousands of square degrees, and hence data analysis methods based on flat sky approximations have become unsatisfactory; procedures which take into account the spherical nature of these observations have become mandatory. These methods are usually based on spherical Fourier analysis, and thus they are described in the frequency domain in terms of the spherical harmonics; this framework, however, can often turn out to be inadequate, due to the lack of localization properties in the real domain. Indeed, real data are typically characterized by huge regions of masked data and/or other features for which localization in the real domain is highly desirable; for this reason, several procedures involving spherical wavelets have become rather popular in astrophysical data analysis, see for instance McEwen et al. (2007), Starck et al. (2006), Donzelli et al. (2012), Fä̈ et al. (200), Marinucci et al. (2008), Pietrobon et al. (2008) and Starck et al. (2010) for a review.

The next decade will probably be characterized by an equally amazing improvement on the quality of observational data: in particular three-dimensional investigation of 
weak gravitational lensing and large scale structure are expected to be implemented by experiments such as Euclid, see for instance Laureijs et al. (2010). These developments clearly motivate the implementation of three-dimensional wavelet systems; to this issue our paper is devoted.

\section{The radial $3 \mathrm{D}$-needlet construction}

Some important efforts have already been spent for the construction of three-dimensional wavelets in an astrophysical context, especially in the last few years, see for instance Lanusse et al. (2012) and Leistedt et al. (2012). In the former reference, wavelets are implemented using a frequency filter on the Fourier-Bessel transform of the three-dimensional field, while in the latter the authors focus on the discretization of the Fourier-Bessel transforms in terms of damped Laguerre polynomials, which allow for an exact quadrature rule.

Our construction is somewhat related to these proposals, and it is based on the astrophysical applications we have in mind Consider for instance an observer located at the centre of a unit ball: we assume that this observer is collecting observations on a set of concentric spheres centred at the origin. At a given resolution level, we also assume that the pixelization on each of these spheres is the same. It is then natural to exploit this implicit radial symmetry, and to view the unit ball as a manifold $M=[0,1] \times \mathbb{S}^{2}$,: the standard spherical Laplacian has then to be modified, implying that the distance between two points on the same spherical shell depends only on the angular part and not on the radius of the shell. The corresponding eigenfunctions have very simple expressions in terms of sines and spherical harmonics: in particular the set of elements given by

$$
u_{\ell, m, n}(r, \vartheta, \phi)=\sin (n r) Y_{\ell, m}(\vartheta, \phi)
$$

provides an orthonormal basis on the space of square-integrable functions over $M$. The system of $3 \mathrm{D}$ radial needlets is then built using the same procedures as for needlets on the sphere (see Narcowich et al. (2006a), Narcowich et al. (2006b)): we compute the convolution of a projection operator by means of a smooth window function $b($.$) , and$ discretize the system by means of an explicitly provided set of cubature points. More precisely, for any resolution level $j$, let the indexes $k$ and $q$ denote respectively the cardinality over the spherical shell and over the radius. Fix a scale parameter $B>1$, and define the radial 3D-needlet as follows:

$$
\Phi_{j, q, k}(r, \vartheta, \varphi)=\sqrt{\lambda_{j, q, k}} \sum_{[\ell, n]_{j}} \sum_{m=-\ell}^{\ell} b\left(\frac{\sqrt{-e_{\ell, n}}}{B^{j}}\right) \bar{u}_{\ell, m, n}\left(\xi_{j, q, k}\right) u_{\ell, m, n}(x),
$$

where $\lambda_{j, q, k}$ and $\xi_{j, q, k}$ are the pixel volume and the pixel center and $e_{\ell, n}$ is the eigenvalue of the Laplacian operator associated to $u_{\ell, m, n}$; finally, observe that $[\ell, n]_{j}$ denotes the pairs of $\ell$ and $n$ s.t. $B^{2(j-1)} \leqslant e_{\ell, n} \leqslant B^{2(j+1)}$. The window function $b(u), u \in \mathbb{R}$ is a positive kernel satisfying three standard properties, namely $b(\cdot)$ has compact support in $[1 / B, B]$, it is infinitely differentiable in $(0, \infty)$ and the following partition of unity property holds: for all $x>B$,

$$
\sum_{j=-\infty}^{\infty} b^{2}\left(\frac{x}{B^{j}}\right)=1
$$

Cubature points and weights are obtained by the tensor products of cubature points on the sphere (as provided by HealPix in Gorski et al. (2005), for instance), and a 
uniform discretization on the radial part, sufficient for exact integration of trigonometric polynomials. Some features of this construction can be illustrated as follows:

(a) Extremely good localization properties hold in both frequency and real domains; these results can be established rigorously by exploiting related arguments on the construction of wavelets for general compact manifolds in Geller et al. (2009), see also Pesenson (2014);

(b) An exact reconstruction formula holds

(c) Computational implementation is simple and effective exploiting existing packages

(d) A direct correspondence holds with standard experimental designs in an astrophysical environment.

\section{References}

Bobin, J., Sureau, F., Paykari P., Rassat, A., Basak, S., \& Starck, J. -L. 2013, A $\& A$, 553, L4

Donzelli, S., Hansen, F. K., Liguori, M., Marinucci, D., \& Matarrese, S. 2012, ApJ, 755, 19

Faÿ, G., Guilloux, F., Betoule, M., Cardoso, J.-F., Delabrouille, J., \& Le Jeune, M. 2008, Phys. Rev. D, D78:083013

Geller, D. \& Mayeli, A. 2009, Math. Z.1, 263

Gorski, K. M. , Hivon, E., Banday, A. J., Wandelt, B. D., Hansen F. K., Reinecke, M., \& Bartelman M. 2005, ApJ, 622

Lanusse, F., Rassat, A., \& Starck, J. L. 2012, A\& A 540, A9

Laureijs, R., Duvet, L., Escudero Sanz, I., Gondoin, P., Lumb, D. H., Oosterbroek T., \& Saavedra Criado G. 2010, The Euclid Mission SPIE Proceedings 7731

Leistedt, B. \& McEwen, J. D. 2012, IEEE Trans. on Sign. Proc. 60, 12

Marinucci, D., Pietrobon, D., Balbi, A., Baldi, P., Cabella, P., Kerkyacharian, G., Natoli, P., Picard, D. \& Vittorio N. (2008 ). 2008, MNRAS, 383, 2

McEwen, J. D., Vielva, P., Wiaux, Y., Barreiro, R. B., Cayón, I., Hobson, M. P., Lasenby, A. N., Martí nez-González, E., \& Sanz, J. L. 2007, J. Fourier Anal. Appl. , 13, 4

Narcowich, F. J., Petrushev, P., \& Ward, J. D. 2006, SIAM Journal of Mathematical Analysis, 38

Narcowich, F. J., Petrushev, P., \& Ward, J. D. 2006, Journal of Functional Analysis, 238, 2

Pesenson, I. Z. 2014, submitted

Pietrobon, D., Amblard, A., Balbi, A., Cabella, P., Cooray, A., \& Marinucci, D. 2008, Phys. Rev. D, D78:103504

Planck Collaboration, 2013, to appear on $A \mathscr{E} A$

Starck, J.-L., Moudden, Y., Abrial P., \& Nguyen, M. 2006,A\&A, 446

Starck, J.-L., Murtagh, F., \& Fadili, J. Sparse Image and Signal Processing: Wavelets, Curvelets, Morphological Diversity (Cambridge University Press) 